\title{
Learning from errors: when a low platelet count in neonate excludes immune thrombocytopenic purpura in mother
}

\author{
Gresikova M \\ Clinic of Pediatric Hematology and Oncology of the School of Medicine, Comenius University, \\ Pediatric Faculty Hospital in Bratislava, Slovakia. gresik@chello.sk
}

\begin{abstract}
This paper reviews the most common causes of thrombocytopenia in the newborn. It mentions few classification schemes that clearly characterize the most common causes, diagnosis and treatment approaches for neonatal thrombocytopenia. Particular attention is paid to inborn macrothrombocytopenia without congenital anomalies. They represent a rare group of diseases, often captured randomly or during routine examinations. An attention is paid on congenital macrothrombocytopenia variants with mutations in the MYH9 gene. If they are not associated with other disorders (deafness, presenile cataracts, nephritis or renal failure), they may be mistakenly diagnosed as the acquired immune thrombocytopenic purpura (ITP). This distinguishing is essential to avoid potentially harmful and unnecessary treatment. The listed case report points to a situation where a detection of the root causes of neonatal thrombocytopenia led to a review of misdiagnosed ITP in the mother. A platelet size evaluation by both an appropriate cell counter and blood film examination is useful for differentiating a heterogeneous group of rare inherited macrothrombocytopenias. A healthy twin supported autosomal dominant inheritance. The results of investigations of twins and mother confirmed the congenital/inherited macrothrombocytopenia from the group of MYH9-related diseases (Tab. 5, Fig. 2, Ref. 21). Full Text in PDF www.elis.sk. Key words: neonatal thrombocytopenia, immune thrombocytopenia, congenital/inherited thrombocytopenia, MYH9-related disease.
\end{abstract}

Thrombocytopenia is the most common haematological abnormality detected in foetus or neonate. The incidence of significant thrombocytopenia at birth (platelet count $<50 \times 10^{9} / \mathrm{L}$ ) is 0.1 to 0.5 $\%$ and the incidence of severe neonatal thrombocytopenia (platelet count $<20 \times 10^{9} / \mathrm{L}$ ) is $0.04 \%$. Neonates with severe thrombocytopenia may have bleeding that leads to lifelong residual defects (e.g., intracranial haemorrhage) or death (1).

Commonly cited causes of neonatal thrombocytopenia (e.g. immunological disordes, congenital and perinatal infections, and disseminated intravascular coagulation) account for only a minority of these early-onset cases. The vast majority of the remaining patients are preterm neonates born following pregnancies complicated by placental insufficiency or foetal hypoxia. In these infants, the incidence of thrombocytopenia is as high as $15 \%$ and it is most severe several days after a delivery. The diagnosis is usually straightforward because the thrombocytopenia is accompanied by other characteristic haematological features seen on routine blood smears (2).

Late-onset neonatal thrombocytopenia (more that 72 hours of age) is mostly caused by late-onset sepsis or necrotizing enterocolitis. Thrombocytopenia is often severe (platelet count $<50 \times 10^{9} / \mathrm{L}$ ), prolonged and prompts treatment by platelet transfusion $(3,4)$.

Clinic of Pediatric Hematology and Oncology of the School of Medicine, Comenius University, Pediatric Faculty Hospital in Bratislava, Slovakia

Address for correspondence: M. Gresikova, MD, DrSc, Clinic of Pediatric Hematology and Oncology of the School of Medicine, Comenius University, Pediatric Faculty Hospital in Bratislava, Limbova 1, SK-833 40 Bratislava, Slovakia.
As majority of other forms of thrombocytopenia would have been resolved by this time, the likely causes of prolonged thrombocytopenia are immune thrombocytopenias, congenital infections, and congenital/ inherited thrombocytopenias. The Table 1 shows the most common causes of neonatal thrombocytopenia by age at presentation (2).

A well neonate presenting with clinical signs of severe thrombocytopenia (e.g. haemorrhage or purpura) is uncommon. However, it represents a neonatal emergency to avoid the potential for severe haemorrhage. In practice, the usual cause is neonatal isolate immune thrombocytopenia. The Table 2 shows the most common causes of neonatal immune thrombocytopenia (1).

Neonatal autoimmune thrombocytopenia (NITP) is an expectable condition. Maternal platelet autoantibodies complicate a number of conditions. The most frequent cause is principally immune thrombocytopenic purpura /ITP/ in a mother, although

Tab. 1. The most common causes of neonatal thrombocytopenia by age at presentation (modified by 2 ).

\begin{tabular}{|c|c|}
\hline \multicolumn{2}{|c|}{ Classification of neonatal thrombocytopenias } \\
\hline $\begin{array}{l}\text { Erly-onset neonatal } \\
(<72 \text { hours of age) }\end{array}$ & $\begin{array}{l}\text { Late-onset neonatal } \\
(>72 \text { hours of age) }\end{array}$ \\
\hline Placental insufficiency & $\mathrm{NEC}$ \\
\hline Perinatal asphyxia and whole body cooling & Congenintal infections \\
\hline Perinatal infections & Autoimmune \\
\hline DIC & Kasabach-Merritt syndrome \\
\hline Alloimmune & Metabolic disease \\
\hline Autoimmune & Congenital/inherited \\
\hline
\end{tabular}


Tab. 2. The most common causes of neonatal immune thrombocytopenia (modified by 1$)$.

\begin{tabular}{|c|c|}
\hline \multicolumn{2}{|c|}{ Causes of neonatal immune thrombocytopenia } \\
\hline $\begin{array}{l}\text { Autoimmune (passive transfer of } \\
\text { plateler antibody) }\end{array}$ & Alloimmune thrombocytopenia \\
\hline a) Maternal ITP & a) Isolated platelet group incompatibility \\
\hline $\begin{array}{l}\text { b) Maternal drug-induced } \\
\text { thrombocytopenia } \\
\text { c) SLE }\end{array}$ & $\begin{array}{l}\text { b) Asssociated with blood group } \\
\text { incompatibility }\end{array}$ \\
\hline
\end{tabular}

a small number of cases will be due to maternal autoimmune disease (systemic lupus erythematosus) (2). This results from the transplacental passage of maternal immunoblobulin G (IgG) autoantibodies into the foetal circulation, with a destruction of foetal platelets. Patients identified by the following criteria should by managed as high-risk patients:

* History of a previously affected infant

* Mother splenectomized for ITP

* Mother with thrombocytopenia (platelets $<100 \times 10^{9} / \mathrm{L}$ ) in the current pregnancy (1)

The nadir of the platelet count in infants with ITP often occurs a few days after delivery. It is, therefore, important that serial counts are obtained during the period of greatest risk for severe thrombocytopenia, the first week of live. Infants with severe thrombocytopenia (platelets $<50 \times 10^{9} / \mathrm{L}$ ) have a $1 \%$ risk for intracranial haemorrhage (ICH) (2).

Neonates with severe thrombocytopenia (platelet count $<30$ x $10^{9} / \mathrm{L}$ ) usually respond promptly to treatment with intravenous immunoglobulins IgG (IVIG) (a dose: $1 \mathrm{~g} / \mathrm{kg}$ /day for 2 days). In urgent situations (e.g. ICH), a combination therapy with highdose IVIG, corticosteroids, and random-donor platelets should be administered.

The platelet counts are commonly rising spontaneously by day 7 , thrombocytopenia may persist in a small number of cases for several weeks $(1,2)$.

Neonatal alloimmune thrombocytopenia (NAIT) should be suspected in thrombocytopenic infants born to mother with a normal platelet count, particularly if infants of successive pregnancies are affected. NAIT is caused by maternal sensitization to foetal platelet antigens inherited from the father. The patophysiology is similar to Rh disease. A number of platelet antigens have been implicated in NAIT. The platelet antigenic system most commonly associated with NAIT has been designated human antigenic system (HPA). In Caucasians, maternal antiplatelet antibodies are most commonly directed against HPA-1a (80\%), HPA- 5b (10\% to 15 $\%$ ) and occasionally anti HPA-3a, anti-HPA-1b and anti-HPA-15 $(5,6)$. In Asian populations, antibodies are more commonly directed against HPA-4. In some cases of NAIT, the causative antibody is directed at low frequency human platelet antigens, or cannot be identified despite a convincing clinical presentation $(2,5)$.

Because these antibodies can interfere with normal platelet aggregation, a qualitative defect may be present in those platelets that are yet not destroyed by the antibody. This functional platelet defect may explain why the incidence of serious bleeding is higher in infants with NAIT than in NITP infants.
Tab. 3. Diagnostic approchach to infant with thombocytopenias with or without anomalies (modified by 1 ).

\begin{tabular}{ll}
\hline \multicolumn{2}{c}{ Infant's physical examination } \\
\hline normal & congenital anomalies \\
\hline NITP & TAR syndrome \\
NAIT & Fancony anemia \\
Drug-induced & Trisomy 13,18 \\
Metabolic disorders & Rubeolla syndrome \\
Congenital thrombocytopenia without & Dyskeratosis congenita \\
anomalies & Kasabach-Merritt syndrome \\
\hline
\end{tabular}

NAIT - neonatal alloimmune thrombocytopenia; NITP - neonatal autoimmune thrombocytopenia; TAR - thrombocytopenia with absent radii.

Tab. 4. Most frequent constitutional disorders with a macrothrombocytopenia $(10,11)$.

Bernard-Soulierov syndrom

Chromosome 11q terminal deletion-related disease (Jacobsen and Paris-Trousseau syndromes)

GATA 1-related disease (dyserythropoietic anemia with macrothrombocytopenia and X-linked thrombocytopenia with thalassemia)

Gray platelet syndrome

Macrothrombocytopenia- MYH 9-related disease (May Hegglin anomaly; Sebastian, Fechtner, and Epstein syndromes)

Phytosterolemia (Mediterranean stomatocytosis and macrothrombocytopenia)

White platelet syndrome

NAIT usually presents in otherwise well term neonates with unexplained bruising and purpura. The platelet count is usually $<30 \times 10^{9} / \mathrm{L}$. Up to $20 \%$ of affected infants will suffer serious bleeding ICH. This occurs in utero in 25 to $50 \%$ of cases and is associated with long-term neurodevelopmental sequelae in $20 \%$ of survivors (7).

The incidence is approximately 1:1000 live births, although approximately $25 \%$ of cases may be clinically silent $(5,6)$. In 50 $\%$ of cases, first-born offspring is affected, suggesting that antigenic exposure can occur during the early course of pregnancy.

Laboratory confirmation is difficult but important with the reference to future pregnancies. A diagnosis of NATP is often inferential. The usual criteria include: normal maternal platelet count and negative history of maternal ITP, and no evidence of systemic disease, infections, malignancy, or haemangioma. The laboratory diagnosis of NAIT is made by demonstrating antiplatelet antibodies (usually anti-HPA-1a antibodies) in maternal serum, which are directed against paternal antigens and by platelet genotyping usually by polymerase chain reaction (6).

Treatment of mildly affected babies is not required, although the platelet count should by monitored for the first 5 days after delivery. By contrast, severely affected infants with NAIT (platelet count $<30 \times 10^{9} / \mathrm{L}$ and/or ICH or other major bleeding) should by promptly transfused with HPA-compatible platelets or washed maternal platelets. If severe thrombocytopenia persist, IVIG is often useful ameliorating the thrombocytopenia until a spontaneous recovery occurs 1 to 6 weeks after birth $(2,5-7)$.

Congenital/inherited thrombocytopenies are a heterogeneous group of rare disorders both dominant and recessive forms of pure thrombocytopenia. Distribution is not uniform due to different clas- 
sification criteria. In majority of neonates, but not in all, there are associated congenital anomalies that are useful in guiding investigation and establishing the diagnosis (Tab. 3) (1). Difficulties in the diagnosis cause mainly trombocytopenia without striking somatic abnormality. Platelet size evaluation by both an appropriate cell counter and blood film examination is useful for differentiating the heterogeneous group of rare disorders, inherited macrothrombocytopenias. This is characterized by abnormal giant platelets, thrombocytopenia and bleeding tendency with variable severity (8). Bone marrow examination demonstrates normal or increased numbers of megakaryocytes (9). The most frequent constitutional disorders with a macrothrombocytopenia are in Table $4(10,11)$.

\section{Case report}

Our patient was a boy, first of binovular twins, in whom at the day of birth a significant thrombocytopenia was found. In the second twin - girl, thrombocytopenia was not confirmed. Twins came from the second, high risk pregnancy (first one was interrupted by abortion). Mother was from the age of 17 years treated for severe thrombocytopenia accidentally trapped. Despite the long term serious platelets decline $<30 \times 10^{9} / \mathrm{L}$ she had no significant bleeding difficulties. She has undergone various therapy within 6 years (IVIG, high dose methyl-prednisolone, prednisone), without a significant effect.

Refractoriness of the disease led to a re-investigation of bone marrow hemopoiesis, without changing the diagnosis. After 2 years of treatment she underwent splenectomy without affecting the platelet amount. At the age of 23 she became pregnant for the second time. During pregnancy, platelet count was critically low (platelet count 3-4 × 10/L), but without bleeding manifestations. She was not treated due to any other disease, she had no handicap (e.g. hearing or visual impairment, kidney disease). In the 35th

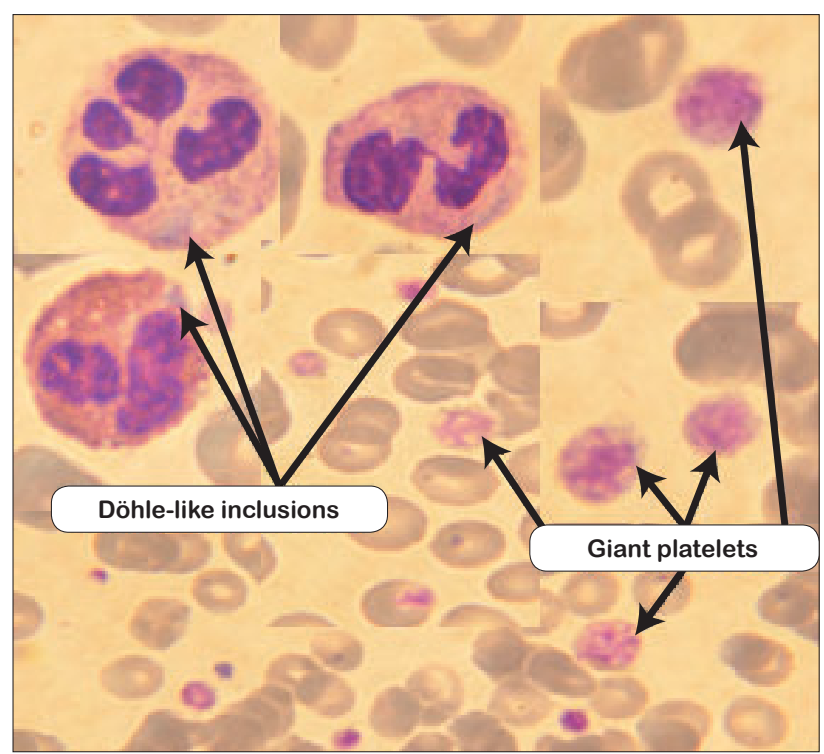

Fig. 1. Peripheral blood smear of a boy: large and giant platelets, basophilic Döhle-like inclusions in leukocytes.

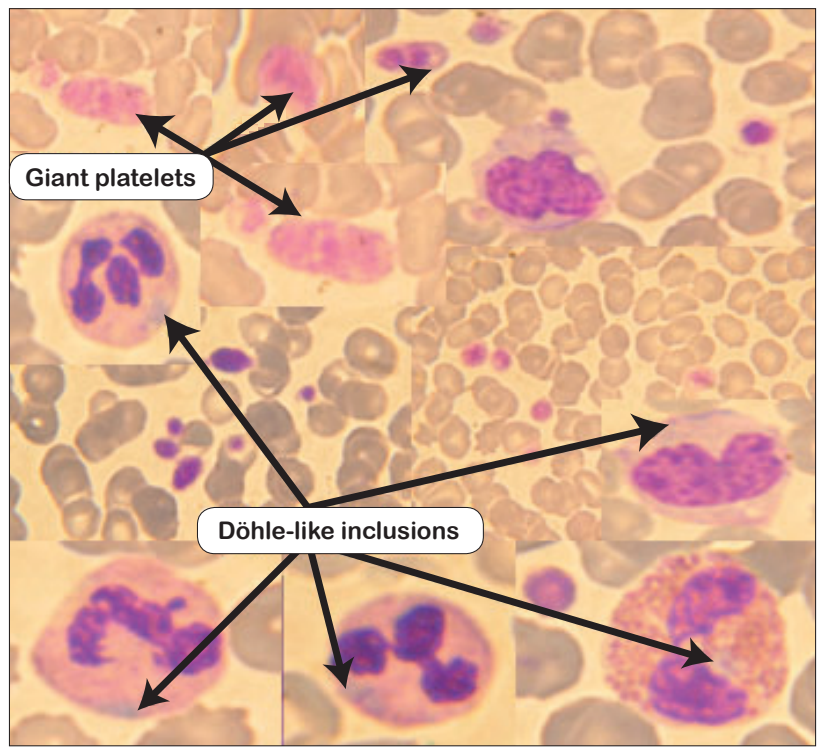

Fig. 2. Peripheral blood smear of a mother: large and giant platelets, basophilic Döhle-like inclusions in leukocytes.

gestational week, after a preparation (combination therapy: IVIG $1 \mathrm{~g} / \mathrm{kg} /$ days for 2 days and corticosteroids and thrombocyte concentrate), pregnancy was terminated by the Caesar section. The mildly immature twins were somatically healthy, without bleeding or other signs of significant adjustment difficulties. Thrombocytopenia was confirmed on the day of delivery, but surprisingly only in a boy (platelet count $41 \times 10^{9} / \mathrm{L}$ ). The girl had normal platelet counts. The boy was treated with IVIG ( $1 \mathrm{~g} / \mathrm{kg} /$ day for 2 days). Monitoring of platelet count in the next three weeks confirmed a nonlinear variation in platelet counts between 43 and $111 \times 10^{9} / \mathrm{L}$. At the age of 8 weeks of life, twins were examined in the paediatric ambulance for the haematological diseases. Girl had normal platelet value repeatedly confirmed. The boy had a mild, clinically asymptomatic thrombocytopenia, which persisted and varied in other months of life (platelet counts from 71 to $127 \times 10^{9} / \mathrm{L}$ ). An unique situation has given rise to questions:

1. Can a mother with severe ITP give birth to twins - one with thrombocytopenia requiring treatment and the second one healthy?

2. What kind of thrombocytopenia has the child, when it persists beyond the neonatal period?

3. Could the mother be incorrectly diagnosed?

The microscopic evaluation of peripheral blood smear of the boy surprisingly confirmed a higher platelet count (platelet count $121 \times 10^{9} / \mathrm{L}$ ) than the count obtained by an automat blood analyzer (platelet count $71 \times 10^{9} / \mathrm{L}$ ). The difference was caused by large populations of giant platelets, which the automat blood analyzer evaluated incorrectly as other blood cells. In the blood smear, anisocytosis of platelets was remarkable with a shift to large forms and giant-forms (about $25 \%$ ). The platelet aggregates were not present in the blood smear and so the EDTA-dependent pseudothrombocytopenia was excluded. In the population of leukocytes (neutrophils, monocytes, eosinophils), basophilic Döhle-like inclusions (90 \% granulocytes) were pres- 
ent (Fig. 1). A coagulogram was appropriate for the age. The results raised a suspicion of inherited macrothrombocytopenia. Mother received a combination therapy with high-dose IVIG, corticosteroids, and transfusion with compatible platelets before delivery. Despite the treatment, an unchanged and critical platelet count persisted, but the patient did not bleed. At the time of examination of the children, the 3-a liner romiplostimom treatment was recommended to the mother.

For a strong suspicion on familiar macrothrombocytopenia, the mother was asked to be examined after the agreement. The microscopic findings were similar to the child: mild thrombocytopenia was confirmed (platelet count $111 \times 10^{9} / \mathrm{L}$ ), with a predominance of large and giant platelets (up to $70 \%$, the "giant platelets" 20 $\%$ ), platelets of appropriate size were a minor population. The automat blood analyzer counted only platelets with physiological size and settled a false thrombocytopenia (platelet count $6 \mathrm{x}$ $\left.10^{9} / \mathrm{L}\right)$. In all granulocytes, the basophilic Döhle-like inclusions were present (Fig. 2). Mother did not suffer in adult from hearing or visual impairment or kidney disease. In mother`s parents, disorder also did not occur.

\section{Discussion}

The fact that the mother with ITP gives birth to twins, of which only one had thrombocytopenia, was very suspicious and could not be explained. The child had no signs of bleeding in the skin or mucous membranes. The choice of treatment of thrombocytopenia in the newborn resulted from the assumption of its deepening in the postnatal period. Mother on the combined treatment of ITP before and after delivery had no bleeding complications and at 6-year history of refractory ITP with severe decrease in platelets she did not overcome an unexpected severe bleeding episode. The second healthy twin unfixed the diagnosis of ITP in the mother.

When doesn't a low platelet count mean ITP? The cases of constitutional/inherited macrothrombocytopenia that have been reported, were mistakes for ithe mmune-mediated thrombocytopenia for years (12). The automated blood cell counters in the routine clinical use usually miss giant platelets and underestimate the mean platelet volume (13). A distinguishing between the acquired (secondary) thrombocytopenia, especially immune thrombocytopenia purpura (ITP), and the inherited (primary) thrombocytopenia is essential to avoid an unnecessary and potentially harmful treatment $(12,14)$. In the patient and his mother, macrothrombocytopenia was present, the healthy girl supported an autosomal dominant inheritance. The presence of basophilic Döhle-like inclusions in leukocytes narrowed suspected myosin heavy chain 9 (MYH9)related platelet disorders (15-21).
There are autosomal dominant disorders caused by the mutations of MYH9 gene. The MYH9 gene encodes the nonmuscle myosin heavy chain 11A (NMMHC-11A), cytoskeletal contractile protein that has been identified in the long arm of chromosome $22(22 q 12.3-q 13.2)(15,17)$. Several mutations in the MYH9 gene lead to premature release of platelets from the bone marrow, macrothrombocytopenia, and cytoplasmic inclusion bodies within leukocyte (19).

This category encompasses a spectrum of independently described disorders: Epstein syndome, Fechner syndrome, May Heggllin anomaly and Sebastian syndrome. There are autosomal dominant disorders caused by mutations of MYH9 (Tab. 5) $(8,14,15)$.

Clinically, patients may present at birth with macrothrombocytopenia and basophilic Döhle-like inclusions in leukocytes. In childhood or adult life, patients may develop senzorineural hearing loss, cataracts, and glomerulonephritis that may progress to severe renal failure. In May Hegglin anomaly or Sebastian syndrome, the patients classically have macrothrombocytopenia with leukocyte inclusions (the difference being only in subtle ultrastructural change in the leukocyte inclusions) (17). Patients with the other associated features were classified as the Fechtner syndrome or Epstein syndrome based on the presence or absence of leukocyte inclusions (Tab. 5). Thrombocytopenia is usually noted incidentally or on routine screening and does not usually cause a symptomatic bleeding diathesis, although a few patients may have severe bleeding. On laboratory evaluation, patients present with variable thrombocytopenia with platelet counts ranging from 10 to $150 \mathrm{x}$ $10 \%$ L. However, macrothrombocytes are always present, but to a variable degree. Platelets larger than red cell can vary from $3 \%$ to $45 \%$ of all platelets. A variable phenotypic expression in this disorder is seen in those with the same mutation and even within the same family. Platelet survival is normal although bone marrow examination sometimes shows an increased number of megacaryocytes $(2,15,20)$.

In the baby within 1 year of clinical following, no organ disability was found. In the mother, only haematological changes were identified, at the age of 24 years she had not proven renal disease or sensory deficit. Similarly, also mother's parents did not suffer from these diseases. Both underwent surgical procedures without bleeding complications. The triad of symptoms: thrombocytopenia, large platelets and characteristic basophilic Döhle-like inclusions in leukocytes, and autosomal dominant inheritance (mother and one of the twins) indicated MYH9-related disease. Most likely, the variant of the disease is so far May Hegglin anomaly or Sebastian syndrome. An accurate classification requires a long-term monitoring and in case of organ involment may lead to a review of disorder in the MYH9-related disease of platelets.

Tab. 5. Four overlapping syndromes of MYH9-related disease $(8,14,15)$.

\begin{tabular}{lccccc}
\hline Type of syndrome & Thrombocytopenia & Döhle inclusions & Nephritis & Hearing loss & Cataract \\
\hline May Hegglin & + & + & - & - & - \\
Sebastian & + & + & - & - & - \\
Fechtner & + & + & + & + & + \\
Epstein & + & - & + & + & + \\
\hline
\end{tabular}




\section{Conclusion}

Pregnant women with ITP are at a $50 \%$ risk for delivering thrombocytopenic infants, whether or not the mother is thrombocytopenic during pregnancy or at the time of delivery (1). Thrombocytopenia in only one child of the twins has led to a review of mother's diagnosis. The distinguishing of inherited thrombocytopenias from immune thrombocytopenia (ITP) can be difficult, and patients are therefore at risk of misdiagnosis and inappropriate treatments. Although it is known that the most common inherited forms of thrombocytopenia without congenital anomalies are characterized by an increased platelet size, the diagnostic power of this feature has never been investigated. The platelet size evaluation by both an appropriate cell counter and blood film examination is useful for the differentiating of inherited macrothrombocytopenias from ITP. This simple approach and accident of thrombocytopenia only in one baby of twins of mothers with ITP have helped to clarify the diagnosis of mother and child and were essential.

\section{References}

1. Neonatal thrombocytopenia. 269-280. In: Lanzkowsky Ph. Manual of pediatric hematology and oncology. London, $4^{\text {th }}$ edition. Elsevier Academic Press, 2005.

2. Roberts IAG, Murray NA. Thrombocytopenia in the Newborn. 943969. In: Michelson AD. Platelets. Birlington, California,London, $2^{\text {th }}$ edition. Elsevier Academic Press, 2007.

3. Kenton AB, O'Donovan D, Cass DL et al. Severe thrombocytopenia predicts outcome in neonates with necrotizing enterocolitis. J Perinatol 2004; 25 (1): 14-20.

4. Kenton AB, Hegemier S, O'Brian Smith $\mathrm{E}$ et al. Platelet transfusions in infants with necrotizing enterocolitis do not lower mortality but may increase. J Perinatol 2004; 25 (1): 173-177.

5. Kroll H, Yates J, Santoso S. Immunization against a low-frequency human platelet alloantigen in fetal alloimmune thrombocytopenia is not a single event: characterization by the combined use of reference DNA and novel allele-specific cell lines expressing recombinant antigens. Transfusion 2005; 45 (3): 353-358.

6. Davoren A, Curtis BR, Aster RH et al. Human platelet antigen-specific alloantibodies implicated in 1162 cases of neonatal alloimmune thrombocytopenia. Transfusion 2004; 44 (8): 1220-1225.

7. Castro Conde JR, Martínez ED, Rodríguez RC et al. CNS siderosis and Dandy-Walker variant after neonatal alloimmune thrombocytopenia. Pediatric Neurology 2005; 32 (5): 346-349.
8. Kunishima S, Saito H. Congenital macrothrombocytopenias. Blood Rev 2006; 20 (2): 111-121.

9. Nurden AT, Nurden P. Inherited thrombocytopenias. Haematologica 2007; 92 (9): 1158-1164.

10. Constitutional hematopoietic disordes. In: Farhi DC: Pathology of bone marrow and blood cells. Baltimore, Philadelphia, $2^{\text {th }}$ edition. Lippincott Williams\&Wilkins, 2009; 51-61.

11. Vannucchi AM, Pancrazzi A, Guglielmelli P et al. Abnormalities of GATA-1 in megakaryocytes from patients with idiopathic myelofibrosis. Am J Pathol 2005; 167 (3): 849-858.

12. Drachman JG. Inherited thrombocytopenia: when a low platelet count does not mean ITP? Blood 2004; 103 (2): 390-398.

13. Gohda F, Uchiumi H, Handa H, Matsushima $T$ et al. Identification of inherited macrothrombocytopenias based on mean platelet volume among patients diagnosed with idiopathic thrombocytopenia. Thromb Res. 2007; 119 (6): 741-746.

14. Noris P, Klersy C, Zecca M, Arcaini L et al. Platelet size distinguishes between inherited macrothrombocytopenias and immune thrombocytopenia. J Thromb Haemost 2009; 7 (12): 2131-2136.

15. Althaus K, Greinacher A. MYH9-related platelet disorders. Semin Thromb Hemost 2009; 35 (2): 189-203.

16. Chen Z, Shivdasani RA. Regulation of platelet biogenesis: insights from the May-Hegglin anomaly and other MYH9-related disorders. J Thromb Haemost 2009; 7, Suppl 1: 272-276.

17. Kunishima S, Yoshinari M, Nishio H et al. Haematological characteristics of MYH9 disorders due to MYH9 R702 mutations. Eur J Haematol 2007; 78 (3): 220-226.

18. Pecci A, Canobbio I, Balduini A et al. Pathogenetic mechanisms of hematological abnormalities of patients with MYH9 mutations. Hum Mol Genet 2005; 14 (21): 3169-3178.

19. Johnson GJ, Leis LA, Krumwiede MD et al. The critical role of myosin IIA in platelet internal contraction. J Thromb Haemost 2007; 5 (7): 1516-1529.

20. Dong F, Sufeng L, Pujol-Moix N et al. Genotype-phenotype correlation in MYH9-related thrombocytopenia. British Br J Haematol 2005; 130 (4): 620-627.

21. Kunishima S, Matsushita T, Hamaguchi M et al. Identification and characterization of the first large deletion of the MYH9 gene associated with MYH9 disorders. Eur J Haematol 2008; 80 (6): 540-544.

Received December 27, 2010. Accepted January 20, 2013. 